\title{
Fabrication of $\mathrm{MnO}_{2}$ Nanocomposite on GO Functionalized with Advanced Electrode Material for Supercapacitors
}

\author{
R. Ranjith Kumar, ${ }^{1}$ S. Thanigaivel, ${ }^{2}$ A. K. Priya $\mathbb{D}^{3},{ }^{3}$ Alagar Karthick $\left(\mathbb{D},{ }^{4,5}\right.$ \\ Chandrabhanu Malla $\mathbb{D}^{6}{ }^{6}$ P. Jayaraman, ${ }^{7}$ M. Muhibbullah $\mathbb{D}^{8},{ }^{8}$ Razan A. Alshgari, \\ and Abdulnasser Mahmoud Karami ${ }^{9}$ \\ ${ }^{1}$ Department of Civil Engineering, SRM Institute of Science and Technology, Delhi NCR Campus, Modinagar, Ghaziabad, \\ 201204 Uttar Pradesh, India \\ ${ }^{2}$ Saveetha School of Engineering, Department of Biotechnology, Saveetha Institute of Medical and Technical Sciences, Thandalam, \\ Chennai, 602105 Tamil Nadu, India \\ ${ }^{3}$ Department of Civil Engineering, KPR Institute of Engineering and Technology, Coimbatore, 641407 Tamil Nadu, India \\ ${ }^{4}$ Renewable Energy Lab, Department of Electrical and Electronics Engineering, KPR Institute of Engineering and Technology, \\ Coimbatore, 641407 Tamil Nadu, India \\ ${ }^{5}$ Departamento de Quimica Organica, Universidad de Cordoba, EdificioMarie Curie (C-3), Ctra Nnal IV-A, Km 396, \\ E14014 Cordoba, Spain \\ ${ }^{6}$ Department of Mechanical Engineering, Radhakrishna Institute of Technology and Engineering, Bhubaneswar, \\ 752057 Odisha, India \\ ${ }^{7}$ Department of Mechanical Engineering, Prathyusha Engineering College, Aranvoyal Kuppam, Tiruvallur, \\ 602025 Tamil Nadu, India \\ ${ }^{8}$ Department of Electrical and Electronic Engineering, Bangladesh University, Dhaka 1207, Bangladesh \\ ${ }^{9}$ Chemistry Department, College of Science, King Saud University, Riyadh 11451, Saudi Arabia
}

Correspondence should be addressed to M. Muhibbullah; m.muhibbullah@bu.edu.bd

Received 10 November 2021; Accepted 24 December 2021; Published 21 January 2022

Academic Editor: Awais Ahmed

Copyright $\odot 2022$ R. Ranjith Kumar et al. This is an open access article distributed under the Creative Commons Attribution License, which permits unrestricted use, distribution, and reproduction in any medium, provided the original work is properly cited.

A new strategy for supercapacitor formation was carried out in the study using electrodes made of graphene oxide (GO) and manganese dioxide $\left(\mathrm{MnO}_{2}\right)$ nanocomposites. To the present knowledge, only a few investigations have been carried out concerning the synthesis of $\mathrm{GO}-\mathrm{MnO}_{2}$-based nanocomposites and their electrochemical properties, with varying mass ratios, as well as the change in electrochemical properties of their components as $\mathrm{MnO}_{2}$ and $\mathrm{GO}$ were tested individually for the enhanced stability and performances. A synthetic method was performed successfully to manufacture $\mathrm{MnO}_{2} / \mathrm{GO}$ nanocomposites. The findings of the present study show that the composites have a lot of potential as an effective conduction property. A composite of graphene oxide supported manganese dioxide nanocomposites fabricated with the simple soft chemical route. As-prepared nanocomposites can be improved in performance by the interactions between $\mathrm{GO}$ and $\mathrm{MnO}_{2}$.

\section{Introduction}

In the last few decades, the importance of nanomaterials was enormously increased due to their unique features and mechanical properties. Various approaches have been implemented to develop a vast number of nanomaterials through the dedicated synthesis methods of top-down and bottom-up methods which are considered as the most common and commercial techniques for nanomaterial synthesis. Nanofragmentation is considered as one of the effective and most promising methods in the field of nanotechnology to generate and tune the nanomaterials into different unique 


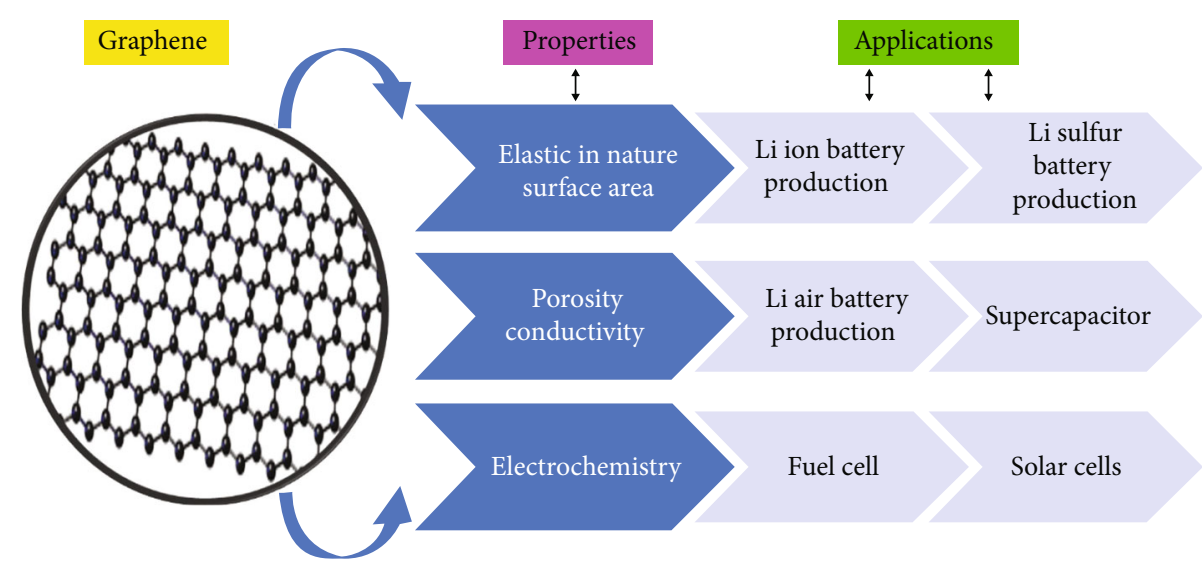

FIgURE 1: Mechanical and electrical properties and their applications of graphene.

nanostructures like nanotubes, nanofibers, and nanorods. This makes them valuable for real-world applications concerning its property which have also been discussed in Figure 1 with the various phenomena. Energy is considered to be one of the most significant needs in today's world, and it is used in various forms $[1,2]$. Nonetheless, rising energy demand causes natural issues and the exhaustion of petroleum derivatives such as fossil fuels. Therefore, a serious examination of energy stockpiling and conversion has gotten a great deal of consideration as far as future innovation advancement. Due to its powerful thickness, long cycle life, and quick charging rate, supercapacitors are increasingly being used to supplement or even replace batteries in a variety of applications $[2,3]$. The $\mathrm{M}_{30}$ electrode has the largest specific capacitance; they can be credited in the form of joined concepts to make $\mathrm{MnO}_{2}$ as a redox potential of pseudocapacitance and reduced graphene oxide (rGO) as an electrical twofold layer capacitance. The $\mathrm{M}_{30}$ electrode's further developed performance could also be attributed to its high graphene carbon content, which helps in diminishing the cationic way into the terminal network that is electrode matrix and bringing down move obstruction which is transfer resistance.

There is a large demand for both conventional and nonconventional energy resources as there is a sudden rise in population and also an increase in living standards. There is a need for nonconventional renewable energy sources due to the increase in greenhouse gas and the depletion of fossil fuels. Therefore, electrochemical energy systems are found to be the alternative for this problem [4]. Recently, electrochemical capacitors like supercapacitors have been introduced over conventional energy stockpiling because of their powerful thickness and energy density when compared to the conventional and recent batteries in Figure 2. They have been used in dc motor drives, robots, electric vehicles, and even UPS (uninterruptable power supply) systems. Due to the charge capacitance, the storage of supercapacitors is subdivided into three different types such as electrical double-layer capacitors (EDLCs), psuedocapacitors, and hybrid or mixed electrodes; recent advancements in the supercapacitance are discussed in Figure 3. The best supercapacitors are the ones that should possess maximum energy

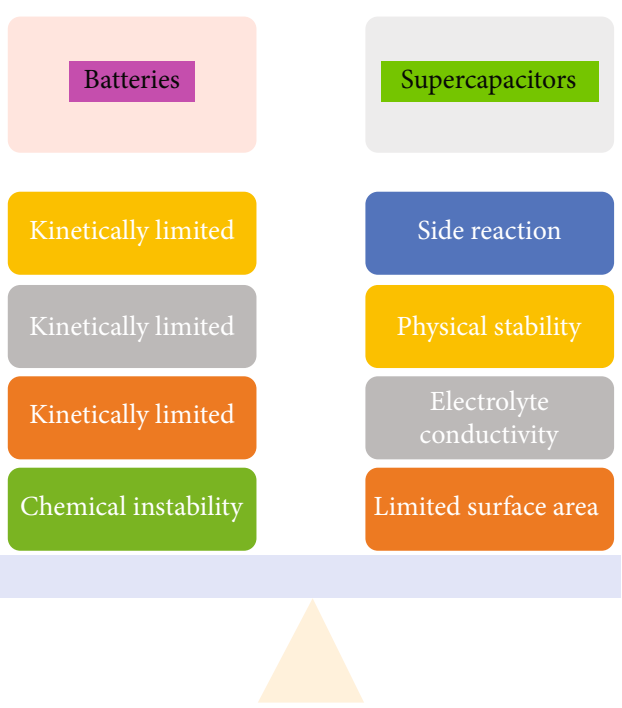

Figure 2: Comparison of batteries' and supercapacitors' performances.

storage capacitance. The supercapacitor capacitance value is decided by two storage principles, which both grant indivisibly to the total capacitance [5].

As an alternative to ruthenium oxide $\left(\mathrm{RuO}_{2}\right)$ (which is widely used in metal oxide supercapacitors), manganese oxide $\left(\mathrm{MnO}_{2}\right)$ offers the advantage in terms of high hypothetical capacitance, cost proficiency, and less toxicity as well as environmental safety $[6,7] . \mathrm{MnO}_{2}$ has been used for lithium batteries, sensors, catalysts, and alkaline $\mathrm{Zn} / \mathrm{MnO}_{2}$ cell applications; under ambient settings, $\mathrm{MnO}_{2}$ is perhaps considered as the most steady manganese oxides, with remarkably strong physical and excellent compound properties. These mixtures' high polymorphism and underlying adaptability have given them a wide scope of uses, including catalysis, biosensors, and energy stockpiling. $\mathrm{MnO}_{2}$ electrodes have set up themselves as a conceivably ideal terminal material for replacing $\mathrm{RuO}_{2}$ in supercapacitors because of their high explicit capacitance, natural similarity, and cost viability [8-11].

The main concerns regarding for advancement of active materials in supercapacitor development were underlying 


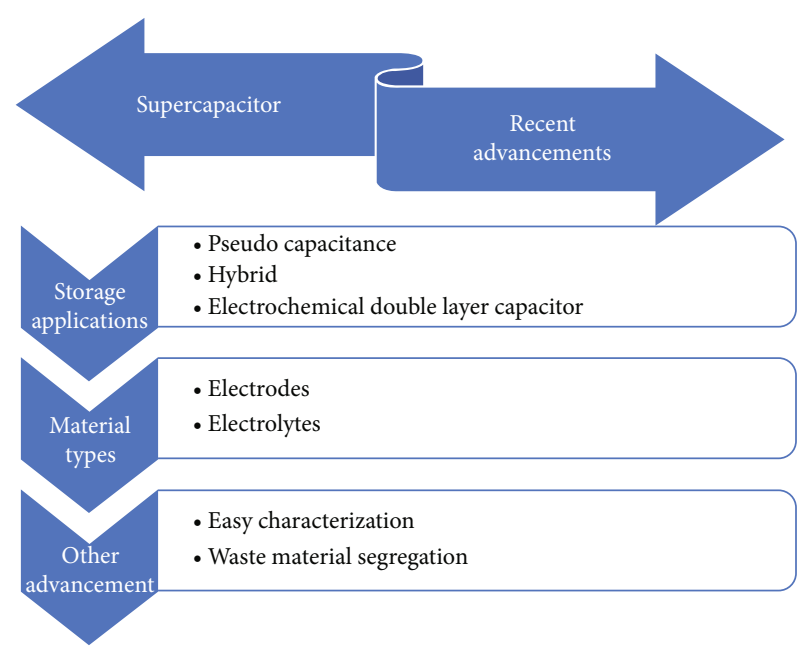

FIgURE 3: Recent advancements in supercapacitor.

adaptability and dependability, quick cation dissemination under high charge-release rates, high reversible capacitance, and natural friendliness. In general, manganese is a change metal component that can exist in different types of stable oxides, viz, $\mathrm{MnO}, \mathrm{Mn}_{3} \mathrm{O}_{4}, \mathrm{Mn}_{2} \mathrm{O}_{3}$, and $\mathrm{MnO}_{2}$, and crystallizes in different types of crystal structures $[12,13]$. Usually, $\mathrm{MnO}_{2}$ is amorphous. Deposition of $\mathrm{Fe}$ does not change the amorphous nature of $\mathrm{MnO}_{2}$ but changes its surface morphology that results in increased porosity, increasing the pseudocapacitive performance of $\mathrm{MnO}_{2}[1,14,15]$. Both active and inert electrodes may be used in high surface area, conductive materials for capacitive supercapacitors that utilize infinitesimal charge partition, or quick, reversible redox responses in the active materials for pseudocapacitance $[16,17]$.

Graphene is a 2D (dimensional) single-layer hexagonal lattice closely packed by $\mathrm{sp}^{2}$ carbon molecules with a carbon-based nanostructure, namely, carbon-carbon distances of $0.142 \mathrm{~nm}$ between the atoms. Over the most recent days, the emergence of graphene-mediated materials is used for the fabrications of graphene-based composites which are made a lot of consideration in the field of science and technology because of their novel properties like high mechanical strength, great thermal and electrical conductivity, innate adaptability, and good optical and electrochemical features. It is the first crystal that is steady at normal temperature conditions, especially at room temperature [17-20].

Since its experimental discovery in 2004 [18], graphene has been utilized in a variety of applications. In light of its uniqueness, it has piqued the interest and imagination of scholars. It has high surface region, great conductivity, and other unique qualities the ease with which it may be functionalized, the mechanical qualities that it has, and as a result, graphene nanosheets have gained a lot of attention. There is a lot of excitement about the possible applications in a variety of technology fields, nanoelectronics, batteries, and supercapacitors, to name a few sensors and field-effect transistors [21-23].

Graphene has high conductivity and surface area that can be considered as an ideal choice for use in supercapacitors because of its capacitance values in the selected mate- rials which are directly proportional to its surface area. As surface area increases, its energy storage value also increases. The capacitance value of graphene materials varies and depends on how they have been stacked. If the surface area of the graphene sheet increases, it can easily be accessible to electrolytes and contributes to the capacitance. So improper stacking of graphene also leads to less capacitance. Hence, avoiding the inner or outer sheet restacking can help graphene in increasing the performance of energy storage. Thus, graphene-based nanomaterials provide an ideal platform to develop various potential materials in the branches of electronics, energy storage devices, and biosensors. The electrons are generally delocalized on either side of the single graphene sheet layer sheet which acts as a barrier and prevents the sheet from restacking; at the same time, these electrons can be useful for identifying other surfaces to bind. Oxidation and shedding of graphite lead to the development of hydrophilic graphite oxide known as graphene oxide. The single-layer oxide of graphene possesses several oxygen species in its structure and aids in various chemical modifications. Hence, numerous graphene-based functional nanomaterials can be generated according to their chemical modifications.

Graphene oxide possesses good mechanical stability and can act as appropriate support for stacking manganese oxide in electroactive materials for supercapacitor applications [24]. The single-layered graphene oxide gives a high surface region for the deposition of $\mathrm{MnO}_{2}$ nanosheets without any restocking and helps in adhesion to other nanostructures. With the high surface region and high stacking effectiveness of the $\mathrm{MnO}_{2}$-GO composite, the particular capacitance value increases [25].

The present study focuses on the development of a $\mathrm{MnO}_{2} \mathrm{GO}$-based supercapacitor that should attain good electric conductivity. $\mathrm{MnO}_{2}$ and $\mathrm{MnO}_{2}-\mathrm{GO}$ composite can be synthesized by a simple and economical electrodeposition method.

\section{Materials and Methodology}

2.1. Synthesis of $\mathrm{MnO}_{2}-\mathrm{GO}$ Nanocomposites. Advanced Hummers and Offeman's method was used to synthesize GO. Approximately 10 milligrams of graphene oxide with $\mathrm{MnO}_{2}$ was added and processed using an ultrasonicator after dispersion, after which the reaction mixture was boiled under the microwave; then, the reaction mixture was mixed into the solution of $10 \mathrm{ml}$ of $\mathrm{FeCl}_{3}$ and $1 \mathrm{M} \mathrm{H}_{2} \mathrm{SO}_{4}$; this solution was added for about 2 hours using the syringe. The sample mixture was then emptied into $20 \mathrm{ml}$ of doubledistilled water added after stirring. Centrifugation was used to obtain $\mathrm{MnO}_{2} / \mathrm{GO}$ nanocomposites, which were then washed several times with water. The overall process has been schematically represented in Figure 4 [26-28].

2.2. Fabrication of $\mathrm{MnO}_{2}$-GO Supercapacitor (SC). $\mathrm{MnO}_{2}$ $\mathrm{GO}$ nanostructures were deposited on current collector substrates by the electrodeposition method by constant potentiostat mode. The stainless steel (SS) substrate was cut and polished into $1 \times 1 \mathrm{~cm}$ and then washed by ultrasonication for $15 \mathrm{~min}$ in deionized water. Electrodeposition was carried 


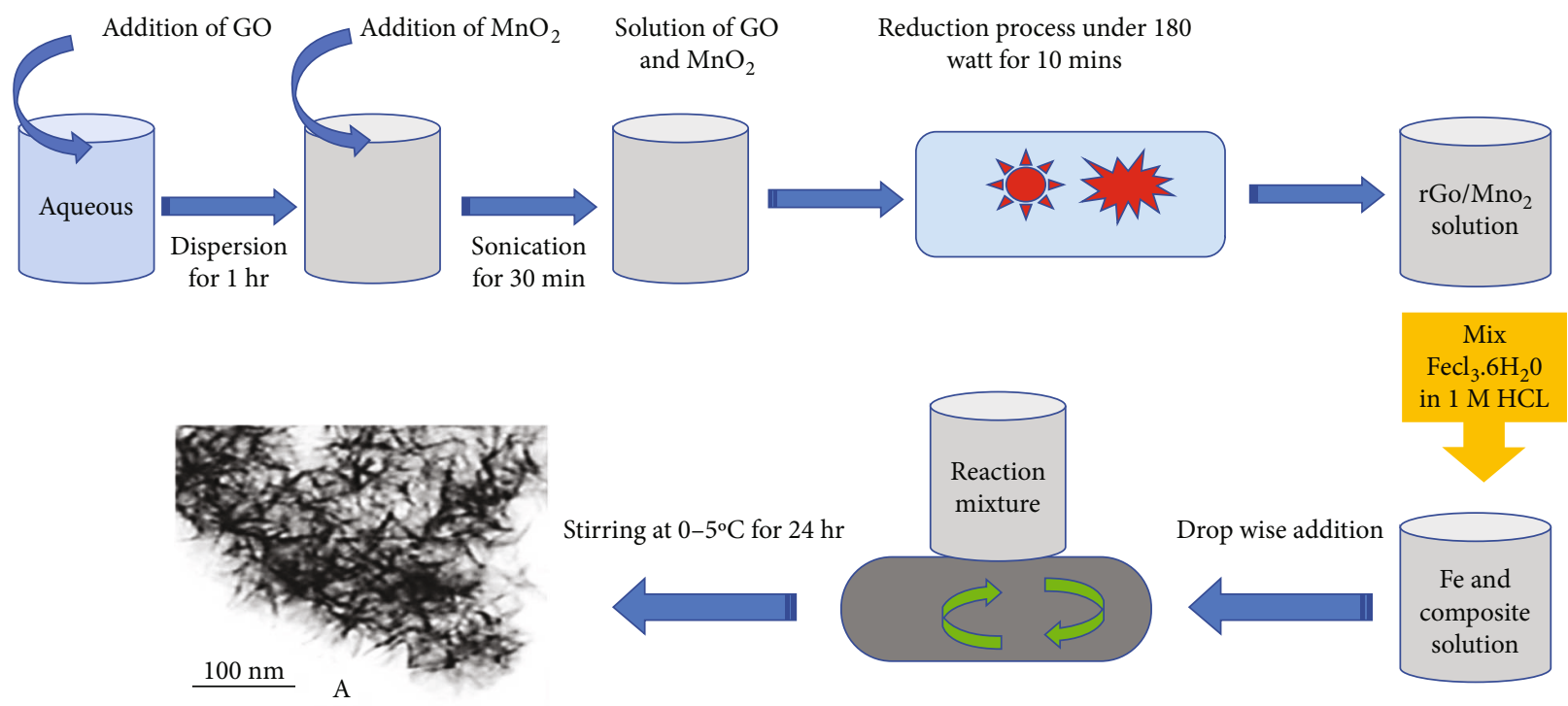

FIGURE 4: Schematic overview of nanocomposite formation under controlled conditions.

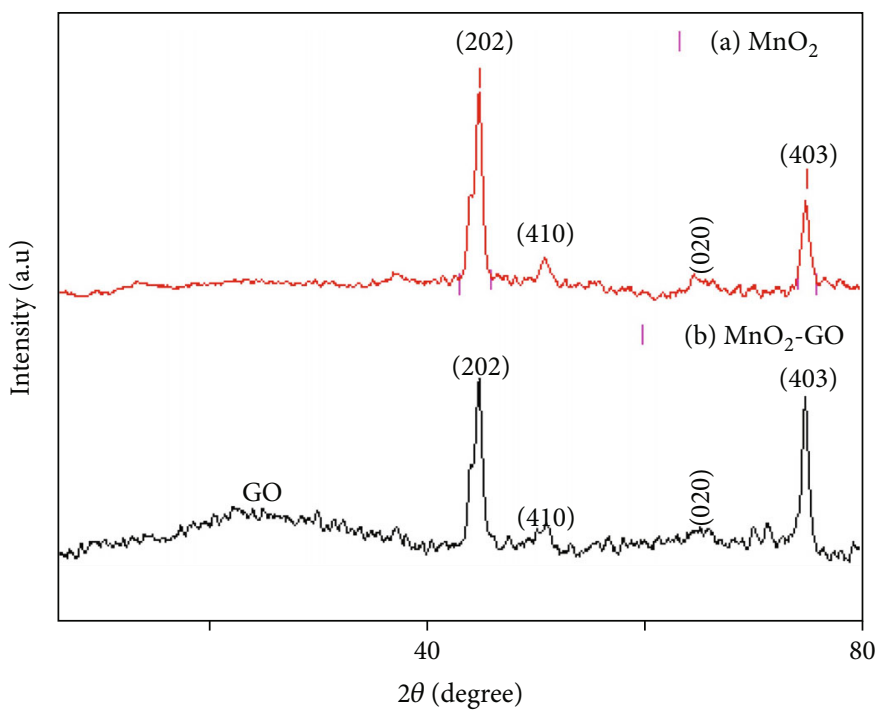

Figure 5: (a) $\mathrm{MnO}_{2}$ and (b) $\mathrm{MnO}_{2}$-GO substrate.

potentiostatically at $0.8 \mathrm{~V}$ for $30 \mathrm{~min}$ using the as-prepared SS substrate considering it as the testing electrode. Platinum and silver/silver chloride terminals were utilized as counter electrodes (reference), individually. The electrolyte was prepared by $20 \mathrm{mg}$ of GO mixed with $25 \mathrm{ml}$ deionized water and dispersed by ultrasonication; then, $0.1 \mathrm{M}$ of $\mathrm{MnSO}_{4}$ arrangement was added. The $\mathrm{pH}$ of the arrangement was made to 10 by adding $1 \mathrm{M} \mathrm{NaOH}$. The resulting films were rinsed in deionized water and dried at room temperature $[14,27,29]$.

2.3. Substrate Characterization. The construction of fabricated nanocomposite thin films was portrayed by powder XRD (Skillet logical XPert Pro MRD diffractometer, Amsterdam, Netherland), worked at $40 \mathrm{kV}$ and $30 \mathrm{~mA}$, and furnished with $\mathrm{Cu}$ radiation at a wavelength of $0.15406 \mathrm{~nm}$. The functional groups of electrodeposited $\mathrm{MnO}_{2}-\mathrm{GO}$ nanocomposites were analyzed by FTIR-ATR. The local type of functional groups in the nanocomposite was recorded in the scope of $3000-500 \mathrm{~cm}^{-1}$ (Fourier transform infrared spectrometer, Shimadzu, Japan).

2.4. Transmission Electron Microscopic Analysis. Transmission electron microscopy was utilized to inspect the morphologies of as-obtained products to determine their shape, size, and dimensions (TEM). All experiments were conducted utilizing a three-anode framework comprising of a functioning electrode made of exposed or modified glassy carbon (GCE, $3 \mathrm{~mm}$ in width), a reference electrode made of saturated calomel (SCE), and an assistant electrode made of platinum wire.

\section{Result and Discussion}

3.1. XRD Analysis. Figure 5 shows the XRD pattern for the $\mathrm{MnO}_{2}$ and $\mathrm{MnO}_{2}-\mathrm{GO}$ deposited films, Figure 5(a) 


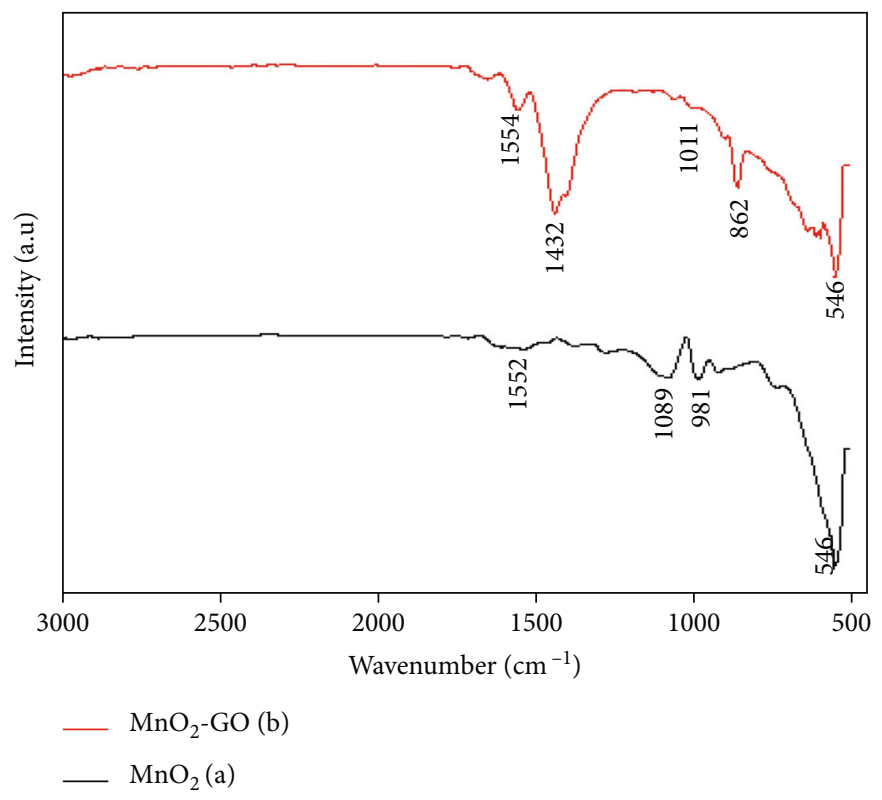

Figure 6: (a) $\mathrm{MnO}_{2}$ and (b) $\mathrm{MnO}_{2}$-GO.

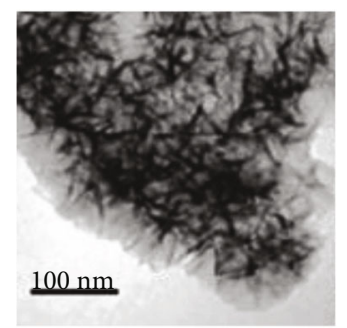

(a)

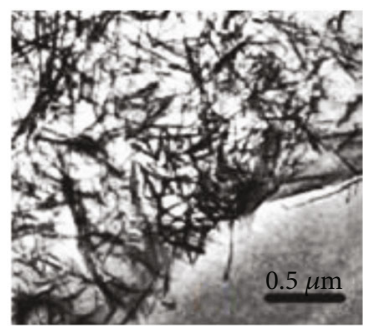

(b)

FIGURE 7: TEM micrograph of nanocomposite morphology: (a) $100 \mathrm{~nm}$ magnification of nanostructure; (b) $500 \mathrm{~nm}$-based magnification of nanostructure.

represents $\mathrm{MnO}_{2}$, and Figure 5(b) represents $\mathrm{MnO}_{2}-\mathrm{GO}$ substrate. All the diffraction peaks for the $\mathrm{MnO}_{2}$ thin film have good agreement with the reference JCPDS (no. 651298), further indexed to orthorhombic $\mathrm{MnO}_{2}$ (orthorhombic crystal structure). The sharp peak tends to the small grain size found as $12 \mathrm{~nm}$ by Scherer's formula. The diffraction pattern of $\mathrm{MnO}_{2}$ - $\mathrm{GO}$ composite also exhibits the same as $\mathrm{MnO}_{2}$ except for the peak around $26^{\circ}$ which is due to the presence of GO. It affirms the presence of $\mathrm{MnO}_{2}$ and GO in the composite. The top at $26^{\circ}$ weak and broad peak is referred from the research article of [24] The average XRD patterns and Raman spectra of chemically synthesized GO- $\mathrm{MnO}_{2}$ nanocomposites were investigated when the $\mathrm{MnO}_{2} / \mathrm{GO}$ feeding ratio was varied. As indicated by the report of the most concentrated GO peak around the 2 theta $10,2^{\circ}$, the (001) reflection is comparative, and because of the introduction of oxygen-containing functional groups on the graphite sheets, the interlayer dividing $(0.87 \mathrm{~nm})$ was a lot bigger than the interlayer of pristine graphite $(0.34 \mathrm{~nm})$.

3.2. FTIR Analysis. FTIR analysis was carried out for SS modified $\mathrm{MnO}_{2}$ and $\mathrm{MnO}_{2}$-GO thin films; as shown in Figure 6, the results show several transmittance peaks for
$\mathrm{MnO}_{2}$ thin films; it represents Figure 6(a) $\mathrm{MnO}_{2}$ and Figure 6(b) $\mathrm{MnO}_{2}$-GO. The strong peak at $546 \mathrm{~cm}^{-1}$ is given to the $\mathrm{Mn}-\mathrm{O}$ stretching vibration of $\mathrm{MnO}_{2}$, and the peaks at 981,1089 , and 1552 are due to the vibration of $\mathrm{O}-\mathrm{O}$ and $\mathrm{OH}$ bending vibrations. For $\mathrm{MnO}_{2}-\mathrm{GO}$ film, the peak at $546 \mathrm{~cm}^{-1}$ for $\mathrm{MnO}_{2}$ and the peaks around $1554 \mathrm{~cm}^{-1} 1432 \mathrm{~cm}^{-1}$ are also found which are attributed to the $\mathrm{C}=\mathrm{C}$ vibrations and $\mathrm{O}-\mathrm{H}$ vibration of graphene oxide. It clearly shows the presence of $\mathrm{MnO}_{2}$ and $\mathrm{GO}$ in the $\mathrm{MnO}_{2}-\mathrm{GO}$ film. The functional group peaks of $\mathrm{MnO}_{2}$ and $\mathrm{GO}$ have been crosschecked from the research publication of [30].

3.3. TEM Analysis. Image of graphene oxide nanosheets were viewed by transmission electron microscopy. The assynthesized GO sheet is discovered to have a smooth surface. With generally high magnification, the manganese oxide nanoparticles were covered with nanosheets of graphene oxide nanoparticles, and more insights concerning the morphology of nanocomposites were investigated, in which GO nanosheets are made out of several layers and the length of each $\mathrm{MnO}_{2}$ nanoparticle is cut formed as slice with scales going from 30 to $50 \mathrm{~nm}$. Moreover, $\mathrm{MnO}_{2} / \mathrm{GO}$ arrangement of nanocomposites can be ultrasonicated in ethanol for 
about an hour with little deficiency of $\mathrm{MnO}_{2}$ from $\mathrm{GO}$, demonstrating that $\mathrm{MnO}_{2}$ adsorption on the outside of $\mathrm{GO}$ is steady. These recently mentioned properties are those of manufactured $\mathrm{MnO}_{2} / \mathrm{GO}$ nanocomposites.

TEM was used to examine $\mathrm{GO} / \mathrm{MnO}_{2}$-based nanocomposites, which were prepared straight from the reaction mixture with no contamination of the surface coating. The surface of the graphene oxide sheet was disordered. The dissolved nanoneedles are less ordered or unbounded as a result of the crystallization process. The sample's morphology was observed as heterostructure nanocomposites in Figure 7 with diameters ranging from 20 to $500 \mathrm{~nm}$. Figure 7(a) shows the resolution of composites at $100 \mathrm{~nm}$ scale range where the clear observations of the nanostructure are observed. In Figure 7(b), the structure of nanocomposites was observed with a $500 \mathrm{~nm}$ scale range for the comparison in the morphology and shapes of the composites, which was further corroborated with [17, $26,31]$. According to the report of [27, 32], the less arranged precursors on GO sheets rapidly evaporated with the development of extra nanoneedles through a dissolution crystallization mechanism as the reaction advanced from 1 to 10 minutes. The morphology of samples with a more extended response time of 30 minutes showed no discernible differences, implying that the crystal formation process was nearly complete after that time $[33,34]$.

In this study, $\mathrm{MnO}_{2}$ /graphene oxide nanocomposites were effectively formed using a simple wet-chemical process. $\mathrm{MnO}_{2}$ is completely covered on the outside of GO nanosheets, according to TEM investigations, and the elements and composition were confirmed by EDX and FT-IR. The content, as well as the crystal structure, was investigated using XRD.

\section{Conclusion}

In this work, pristine $\mathrm{MnO}_{2}$ and $\mathrm{MnO}_{2}$ - $\mathrm{GO}$ composites were successfully coated on SS by the electrodeposition method. The obtained coatings were homogeneous and showed a very good adhesion on the metal substrate. The coated substrates were characterized using XRD and FTIR. The primary properties were examined utilizing the X-beam diffraction method. From the outcomes, we discovered the orthorhombic structure of $\mathrm{MnO}_{2}$ with a molecule size around $12 \mathrm{~nm}$. Besides, we conclude with the presence of $\mathrm{MnO}_{2}$ and graphene sheets in the composite. FTIR spectrum confirmed the presence of $\mathrm{Mn}-\mathrm{O}$ stretching vibration and bending vibration of graphene oxide in the composite.

Graphene has a distinct chemical makeup and a wide range of properties, including exceptional electrical, optical, thermal, and mechanical capabilities. To meet the increased demand for thin-film processing, composite-mediated integration has evolved in recent technologies, and a number of synthetic techniques, including chemical and physical methodologies, have been created for device integration as well. Graphene has been composited with polymers, semiconductor nanoparticles, metals, metal oxides, sulphides, alloys, carbon nanotubes, organic molecules, and other materials. The enhancement of the properties of these composited materials is based not only on the individual components but also on their interaction.

In this study, authors are experiencing the advantage of making novel and an innovative supercapacitor (SC) which was made out of nanocomposites, which had excellent advantages over other conventional capacitors and other electronic devices. The advantages include the following: they are most commonly used in electric hybrid vehicles, military types of machinery, communication devices for uninterrupted power supply in mobiles, and laser devices.

There are certain challenges to overcome; the supercapacitors are cost-effective and highly dense in energy. Recently, many initiatives have been started to overcome the problems associated with the low energy density developed by the SCs which are developed from nanostructure-mediated electrodes with enhanced capacitance. Higher operating voltages can help you save money on your energy bills. The energy density increases as the working potential window widens, needing less material, separator, and electrolyte to store the same amount of energy. A high cell voltage means that fewer cells must be connected in series to form a specific voltage system, reducing the load on external voltage-balance circuits, which are now widely utilized. Last but not least, electrode materials for SCs have sparked a lot of attention in recent years as a way to create cost-effective and highperforming energy storage devices. Waste materials are a cost-effective way to make supercapacitor electrodes for this purpose, albeit they still need a lot of work (fabrication methods, electrolyte selection, etc.) before they can be sold as raw electrode material in the commercial market.

\section{Data Availability}

The data used to support the findings of this study are included within the article.

\section{Conflicts of Interest}

The authors declare no potential conflict of interest.

\section{Acknowledgments}

The authors are grateful to the Saveetha School of Engineering, Saveetha Institute of Medical and Technical Science (also known as Saveetha University), and SRM University, Ghaziabad, for providing us with all of the resources we needed to complete our study. Alagar Karthick gratefully acknowledges group FQM-383 from Universidad de Cordoba, Spain, for the provision of an honorary visiting research position in the group. This work was funded by the Researchers Supporting Project (Number RSP-2021/ 265), King Saud University, Riyadh, Saudi Arabia.

\section{References}

[1] D. P. Dubal, W. B. Kim, and C. D. Lokhande, "Galvanostatically deposited Fe: $\mathrm{MnO}_{2}$ electrodes for supercapacitor application," Journal of Physics and Chemistry of Solids, vol. 73, no. 1, pp. 18-24, 2012. 
[2] Y. Liu, D. Yan, Y. Li et al., "Manganese dioxide nanosheet arrays grown on graphene oxide as an advanced electrode material for supercapacitors," Electrochimica Acta, vol. 117, pp. 528-533, 2014.

[3] Suhasini, "Effect of deposition method and the surfactant on high capacitance of electrochemically deposited $\mathrm{MnO}_{2}$ on stainless steel substrate," Journal of Electroanalytical Chemistry, vol. 690, pp. 13-18, 2013.

[4] T. Yousefi, A. N. Golikand, M. H. Mashhadizadeh, and M. Aghazadeh, "Template-free synthesis of $\mathrm{MnO}_{2}$ nanowires with secondary flower like structure: characterization and supercapacitor behavior studies," Current Applied Physics, vol. 12, no. 1, pp. 193-198, 2012.

[5] B. E. Conway, Electrochemical Supercapacitors: Scientific Fundamentals and Technological Applications, Springer Science \& Business Media, 2013.

[6] H. Yang, J. Jiang, W. Zhou et al., "Influences of graphene oxide support on the electrochemical performances of graphene oxide-MnO 2 nanocomposites," Nanoscale Research Letters, vol. 6, no. 1, p. 531, 2011.

[7] Z. Li, J. Wang, X. Liu, S. Liu, J. Ou, and S. Yang, "Electrostatic layer-by-layer self-assembly multilayer films based on graphene and manganese dioxide sheets as novel electrode materials for supercapacitors," Journal of Materials Chemistry, vol. 21, no. 10, pp. 3397-3403, 2011.

[8] Z. Li, Y. Mi, X. Liu, S. Liu, S. Yang, and J. Wang, "Flexible graphene/MnO 2 composite papers for supercapacitor electrodes," Journal of Materials Chemistry, vol. 21, no. 38, pp. 14706-14711, 2011.

[9] J. Zhang, J. Jiang, and X. S. Zhao, "Synthesis and capacitive properties of manganese oxide nanosheets dispersed on functionalized graphene sheets," The Journal of Physical Chemistry C, vol. 115, no. 14, pp. 6448-6454, 2011.

[10] K. Q. Ding, "Cyclic voltammetrically prepared copperdecorated $\mathrm{MnO}_{2}$ and its electrocatalysis for oxygen reduction reaction (ORR)," International Journal of Electrochemical Science, vol. 5, pp. 72-87, 2009.

[11] L. Zhang, R. Jamal, Q. Zhao, M. Wang, and T. Abdiryim, "Preparation of PEDOT/GO, PEDOT/MnO 2 , and PEDOT/ $\mathrm{GO} / \mathrm{MnO}_{2}$ nanocomposites and their application in catalytic degradation of methylene blue," Nanoscale Research Letters, vol. 10, no. 1, pp. 1-9, 2015.

[12] S. Chen, J. Zhu, X. Wu, Q. Han, and X. Wang, "Graphene Oxide-MnO2Nanocomposites for supercapacitors," ACS Nano, vol. 4, no. 5, pp. 2822-2830, 2010.

[13] A. L. M. Reddy, F. E. Amitha, I. Jafri, and S. Ramaprabhu, "Asymmetric flexible supercapacitor stack," Nanoscale Research Letters, vol. 3, no. 4, pp. 145-151, 2008.

[14] J. Lei, X. Lu, W. Wang et al., "Fabrication of $\mathrm{MnO}{ }_{2} /$ graphene oxide composite nanosheets and their application in hydrazine detection," RSC Advances, vol. 2, no. 6, pp. 2541-2544, 2012.

[15] X. Yang, W. Tang, Q. Feng, and K. Ooi, "Single crystal growth of birnessite-and hollandite-type manganese oxides by a flux method," Crystal Growth \& Design, vol. 3, no. 3, pp. 409415, 2003.

[16] D. Zheng, Z. Yin, W. Zhang, X. Tan, and S. Sun, "Novel branched $\gamma$-MnOOH and $\beta$-MnO2Multipod nanostructures," Crystal Growth \& Design, vol. 6, no. 8, pp. 1733-1735, 2006.

[17] M. Xu, L. Kong, W. Zhou, and H. Li, "Hydrothermal synthesis and pseudocapacitance properties of $\alpha$-MnO2Hollow spheres and hollow urchins," The Journal of Physical Chemistry C, vol. 111, no. 51, pp. 19141-19147, 2007.

[18] S. Devaraj and N. Munichandraiah, "Effect of crystallographic structure of $\mathrm{MnO} 2 \mathrm{on}$ its electrochemical capacitance properties," The Journal of Physical Chemistry C, vol. 112, no. 11, pp. 4406-4417, 2008.

[19] M. Toupin, T. Brousse, and D. Bélanger, "Influence of microstucture on the charge storage properties of chemically synthesized manganese dioxide," Chemistry of Materials, vol. 14, no. 9, pp. 3946-3952, 2002.

[20] Q. Li, J. B. Olson, and R. M. Penner, "Nanocrystalline $\alpha$ MnO2Nanowires by electrochemical step-edge decoration," Chemistry of Materials, vol. 16, no. 18, pp. 3402-3405, 2004.

[21] R. Kötz and M. J. E. A. Carlen, "Principles and applications of electrochemical capacitors," Electrochimica Acta, vol. 45, no. 15-16, pp. 2483-2498, 2000.

[22] M. Kaempgen, C. K. Chan, J. Ma, Y. Cui, and G. Gruner, "Printable thin film supercapacitors using single-walled carbon nanotubes," Nano Letters, vol. 9, no. 5, pp. 1872-1876, 2009.

[23] Q. Wang, Z. H. Wen, and J. H. Li, "A hybrid supercapacitor fabricated with a carbon nanotube cathode and a $\mathrm{TiO}_{2}-\mathrm{B}$ nanowire anode," Advanced Functional Materials, vol. 16, no. 16, pp. 2141-2146, 2006.

[24] P. Simon and Y. Gogotsi, "Materials for electrochemical capacitors," Nanoscience and technology: a collection of reviews from Nature journals, pp. 320-329, 2009.

[25] C. Xu, X. Wang, J. Zhu, X. Yang, and L. Lu, "Deposition of $\mathrm{Co3O}_{4}$ nanoparticles onto exfoliated graphite oxide sheets," Journal of Materials Chemistry, vol. 18, no. 46, pp. 5625-5629, 2008.

[26] S. Chen, J. Zhu, Q. Han, Z. Zheng, Y. Yang, and X. Wang, "Shape-controlled synthesis of one-dimensional MnO2via a facile quick-precipitation procedure and its electrochemical properties," Crystal Growth \& Design, vol. 9, no. 10, pp. 4356-4361, 2009.

[27] Z. Chen, I. Santoso, R. Wang et al., "Surface transfer hole doping of epitaxial graphene using $\mathrm{MoO}_{3}$ thin film," Applied Physics Letters, vol. 96, no. 21, article 213104, 2010.

[28] C. Berger, Z. Song, T. Li et al., "Ultrathin epitaxial graphite: 2D electron gas properties and a route toward graphene-based nanoelectronics," The Journal of Physical Chemistry B, vol. 108, no. 52, pp. 19912-19916, 2004.

[29] Y. Wang, Z. Shi, Y. Huang et al., "Supercapacitor devices based on graphene materials," The Journal of Physical Chemistry C, vol. 113, no. 30, pp. 13103-13107, 2009.

[30] E. Yoo, J. Kim, E. Hosono, H. S. Zhou, T. Kudo, and I. Honma, "Large reversible Li storage of graphene nanosheet families for use in rechargeable lithium ion batteries," Nano Letters, vol. 8, no. 8, pp. 2277-2282, 2008.

[31] F. Schedin, A. K. Geim, S. V. Morozov et al., "Detection of individual gas molecules adsorbed on graphene," Nature Materials, vol. 6, no. 9, pp. 652-655, 2007.

[32] W. Yang, Z. Gao, J. Wang et al., "Synthesis of reduced graphene nanosheet/urchin-like manganese dioxide composite and high performance as supercapacitor electrode," Electrochimica Acta, vol. 69, pp. 112-119, 2012.

[33] L. Wang, Y. Zheng, S. Chen et al., "Three-dimensional kenaf stem-derived porous carbon/ $\mathrm{MnO}_{2}$ for high-performance supercapacitors," Electrochimica Acta, vol. 135, pp. 380-387, 2014.

[34] W. Wei, X. Cui, W. Chen, and D. G. Ivey, "Manganese oxidebased materials as electrochemical supercapacitor electrodes," Chemical Society Reviews, vol. 40, no. 3, pp. 1697-1721, 2011. 